\title{
Confusion, respiratory rate, shock index (CRSI-65) score in the emergency department triage may be a new severity scoring method for community-acquired pneumonia
}

Beliz O. Tekten, MD, Tugba A. Temrel, MD, Serkan Sahin, MD.

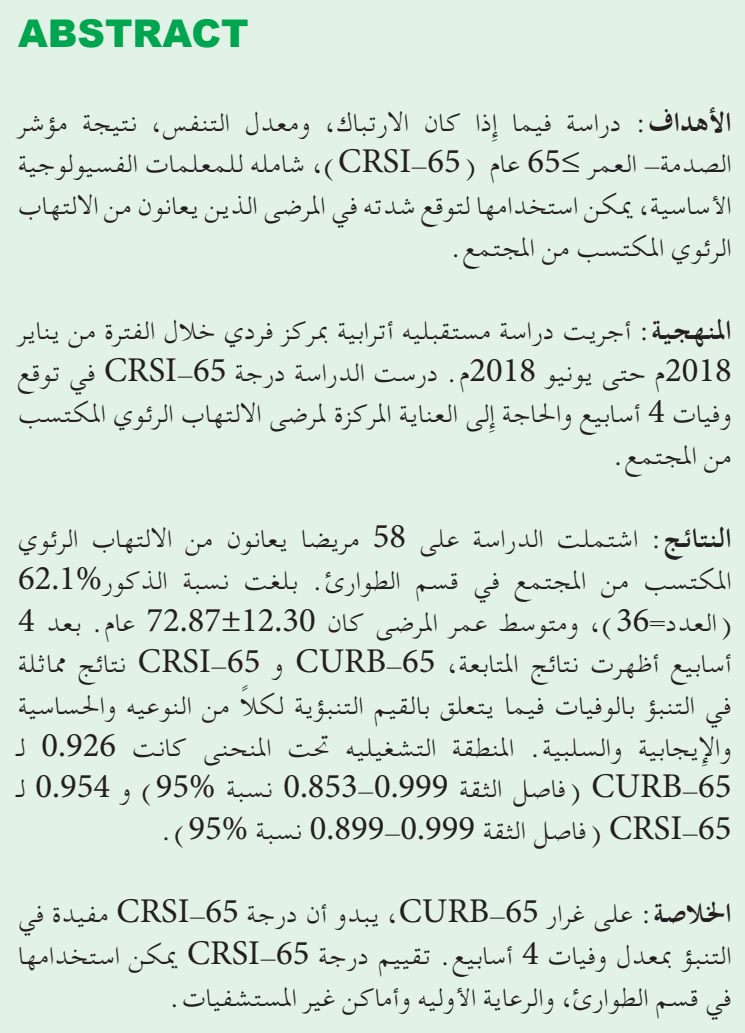

Objectives: To investigate whether confusion, respiratory rate, shock index-age $\geq 65$ years (CRSI-65) score, consisting of basic physiological parameters, can be used for severity prediction in patients with community-acquired pneumonia.

Methods: This is a prospective cohort and singlecenter study conducted in Bolu Abant Izzet Baysal University Hospital, Bolu, Turkey between January 2018 and June 2018. The study investigated CRSI-65 score in predicting 4-week mortality and the need for intensive care for patients with community-acquired pneumonia.

Results: A total of 58 patients with communityacquired pneumonia admitted to the emergency department were included in this study. Of the patients, $62.1 \%$ were males $(n=36)$, and the mean age of the patients was $72.87 \pm 12.30$ years. After 4 weeks of follow-up, CURB-65 and CRSI-65 scores showed similar results in predicting mortality with respect to specificity, sensitivity, and positive and negative predictive values. Area under the receiver operating characteristic curve was 0.926 for the CURB-65 (95\% confidence interval [CI] 0.853-0.999) and 0.954 for the CRSI-65 (95\% CI 0.899-0.999).

Conclusion: Similar to the CURB-65 score, the CRSI-65 score appears to be useful in predicting 4-week mortality. The evaluation of CRSI-65 score can be used in emergency department triage, primary care, and non-hospital settings.

Keywords: CURB-65, pneumonia, emergency service

Saudi Med J 2020; Vol. 41 (5): 473-478 doi: 10.15537/smj.2020.5.25069

From the Department of Emergency Medicine (Tekten), Bolu Abant Izzet Baysal University Faculty of Medicine, Bolu; from the Department of Emergency Medicine (Temrel), Faculty of Medicine, University of Health Sciences, and from the Department of Emergency Medicine (Sahin), Gulhane Training and Research Hospital, University of Health Sciences, Ankara, Turkey.

Received 21st November 2019. Accepted 9th April 2020.

Address correspondence and reprint request to: Dr. Serkan Sabin, Department of Emergency Medicine, Gulhane Training and Research Hospital, University of Health Sciences, Ankara, Turkey. E-mail: raserkan@hotmail.com

ORCID ID: https://orcid.org/0000-0001-6671-1081

Disclosure. Authors have no conflict of interests, and the work was not supported or funded by any drug company. 
C ommunity-acquired pneumonia (CAP) is a lower respiratory tract infection caused by pathogens acquired from sources other than health institutions and represents a serious source of mortality and morbidity. The disease is common worldwide, occurs in almost all age groups, and is a major cause of mortality. ${ }^{1,2}$ Emergency services are the first place of admission for patients with severe CAP as in many diseases. The severity of CAP in such patients is one of the reasons for the unexpected transfer of patients to the intensive care unit (ICU) in the emergency department. Delayed ICU admission has been shown to produce poorer outcomes compared with early ICU admission. ${ }^{3}$ This shows the prognostic importance of early risk identification. Confusion, serum urea nitrogen level, respiratory rate, blood pressure and age $\geq 65$ years (CURB-65). is a well-established scoring system and is recommended by different guidelines for the assessment of clinical risk in patients with CAP. ${ }^{4-6}$ The major disadvantage of the CURB-65 scoring system is that urea level measurement is necessary to evaluate patients' score. This makes it difficult for the CURB-65 to be used for CAP risk scoring in the emergency department triage, in outpatients and in out-of-hospital settings. Moreover, blood pressure, one of the components of CURB-65, may cause false negatives in hypertensive patients. A scoring system most similar to the confusion, respiratory rate, shock index-age $\geq 65$ years (CRSI-65) score was first reported by Curtain et $\mathrm{al}^{7}$ as the CRSI score without the age parameter. Confusion, respiratory rate, shock index-age $\geq 65$ years score is an acronym comprising confusion $(\mathrm{C})$, respiratory rate per minute $(\mathrm{R})$, shock index (SI), and age parameters. The shock index is calculated by proportioning the heart rate to systolic blood pressure. Its normal range is considered to be $0.5-0.7$. It is a scoring system used to demonstrate hemodynamic stabilization. SI $\geq 1$ is significant in predicting the need for urgent treatment. It is a better indicator than systolic blood pressure and heart rate alone in the evaluation of hemodynamics. ${ }^{8}$ These parameters can be easily evaluated in every in-hospital and out-of-hospital settings. Due to the limitations in evaluating the CURB-65 score, different versions of the CURB-65 score have been previously discussed in the literature. ${ }^{1,7,9,10}$ In this single-center pilot study, the CRSI-65 score, which comprises entirely clinical and physiological parameters, to predict CAP mortality and the need for intensive care was investigated.

Methods. This study was conducted in Bolu Abant Izzet Baysal University Hospital, Bolu, Turkey with 320 service beds and 50 intensive care beds. The annual patient burden of the emergency department is 73,000 patients. This was a prospective cohort, singlecenter pilot study conducted between January 2018 and June 2018. A total of 58 patients who met the inclusion criteria during this period were included in the study. The patients were followed up for 4 weeks after their admission to the emergency department. During this period, outpatients were followed up via telephone, while inpatients were followed up on the basis of hospital records. Primary outcome was defined as admission to the ICU and 4-week mortality. Ethics committee approval for the study was obtained from the institution (Ethics committee approval no: 2018/185).

Patients over 18 years of age who had new infiltration consistent with pneumonia in their chest $\mathrm{x}$-ray and consistent clinical findings (namely, coughing, phlegm, dyspnea, and pleuritic chest pain, with or without fever) were included in the study. Factors that may affect the agent of pneumonia and factors that may affect calculated scoring system was excluded. Patients with terminal disease or malignancy, receiving chemotherapy, receiving corticosteroids or immunosuppressive therapy, with malnutrition or cachexia, or suspected of having nosocomial infection were excluded from the study.

Pre-hypothesis power analysis with alpha 0.05 in 95\% confidence interval, sample size was calculated as 158 patients in each group, minimum sample size was 95 patients. ${ }^{11}$ However, only 58 patients who met the inclusion criteria were included in the study. In the post-hoc power analysis at alpha 0.05 and $95 \% \mathrm{CI}$, the power was evaluated as $87.90 \%$.

All procedures that contributed to this study complied with the ethical standards of the relevant national and institutional committees on human experimentation and with the Helsinki Declaration of 1975, as revised in 2008. Approval for the study was granted by the Bolu Izzet Baysal University Institutional Ethics Committee, and written informed consent was obtained from all participants.

The CURB-65 score of each patient included in the study was evaluated as described in previous studies. Consistent with the previous studies on the CAP severity index, patients with a risk score of $\geq 3$ were considered to have severe CAP. ${ }^{11,12}$

For the CRSI-65 score, the urea parameter in the CURB-65 score was removed and the SI, which is the ratio of heart rate to blood pressure, was used instead of the blood pressure parameter. If the patient's SI was $\geq 1$, the patient was given one point for high risk. ${ }^{1,10}$ Other components of the CRSI-65 score-confusion, respiratory rate, and 65 years of age were evaluated in a 
similar manner as that of the CURB-65 score. Thus, the CRSI-65 score was evaluated between 0-4 points. Using the method described by Lim et $\mathrm{al}^{12}$ to investigate the specificity and sensitivity of the CRSI-65 score, a score of $\geq 3$ (as the risk score) was considered as severe CAP for both the CRSI-65 score and the CURB-65 score.

Statistical analysis. We analyzed the data using the Statistical Package for Social Sciences Version 20.0 (IBM Inc., Chicago, IL, USA). Descriptive statistics of the variables were calculated. Categorical variables were expressed as frequency and percentage, whereas continuous numerical data were expressed as mean and standard deviation. The normality analysis of numerical variables was performed using the Kolmogorov-Smirnov test. The Monte Carlo corrected Chi-square method was used to determine the relationships between categorical variables. Receiver operating characteristic analysis was performed, and the areas under the curve were calculated to identify the success of the measurements performed for the re-evaluated indexes and the success of the indexes in predicting intensive care admission and mortality. The results were visualized using graphs. Type-I error rate was taken as $5 \%$ in the entire study, and $p<0.05$ was considered statistically significant. Scoring systems of agreement was calculated with Kappa Measure of Agreement test. The McNemar test was performed between the severity of the 2 systems and added to manuscript.
Results. Of the 58 patients, $62.1 \%$ were male $(\mathrm{n}=36)$. The mean age of the patients was $72.87 \pm 12.30$ years. Mean respiratory rate of the study group was $24.18 \pm$ $5.75 \mathrm{~min}$, whereas its mean systolic blood pressure was $120.94 \pm 25.59 \mathrm{~mm} \mathrm{Hg}$, mean diastolic blood pressure was $72.39 \mathrm{~mm} \mathrm{Hg} \pm 13.58$, pulse $98.15 \pm 17.58$ beats/ $\mathrm{min}$, urea level $7.96 \pm 4.26 \mathrm{mg} / \mathrm{dL}$, and SI was $0.84 \pm$ 0.36 . The ratio of severe CAP cases was $20.6 \%(n=12)$ according to the CURB-65 and $15.5 \%(\mathrm{n}=9)$ according to the CRSI-65 scores. During the 4-week follow-up, mortality occurred in $6.9 \%(n=4)$ patients and $12.1 \%$ $(n=7)$ patients were admitted to the ICU. The rate of hospitalization was $70.7 \%(\mathrm{n}=41)$ in followed patients.

Table 1 shows the characteristics of the subgroups formed according to the severity of CAP, based on the CURB-65 and CRSI-65 scores. There was no significant difference between the severity of CRSI and CURB systems $(p=0.453)$.

Tables 2 \& 3 show the specificity, sensitivity, and positive and negative predictive values of the CURB-65 and CRSI-65 indexes in predicting the 4-week need for intensive care and 4-week mortality of the study group. Receiver operating characteristic analysis revealed that the CRSI-65 score had a diagnostic value in predicting 4-week mortality and need for intensive care in patients with CAP. Receiver operating characteristic curve values (95\% CI) of the CRSI-65 score in predicting 4-week intensive care need was AUC 0.737 (0.506-0.968,

Table 1 - Basic characteristics of the study group according to CURB-65 and CRSI-65 scores.

\begin{tabular}{|c|c|c|c|c|c|c|}
\hline \multirow[t]{2}{*}{ Scores } & \multicolumn{2}{|c|}{ CURB-65 } & \multicolumn{4}{|c|}{ CRSI-65 } \\
\hline & $\begin{array}{c}\text { Non severe }(<3) \\
\quad(n=46)\end{array}$ & $\begin{array}{l}\text { Severe }(\geq 3) \\
\quad(n=12)\end{array}$ & $P$-value & $\begin{array}{c}\text { Non severe }(<3) \\
\quad(n=49)\end{array}$ & $\begin{array}{l}\text { Severe }(\geq 3) \\
\quad(n=9)\end{array}$ & $P$-value \\
\hline Gender (male) (\%) & $29(63.0)$ & $7(58.3)$ & 0.765 & $32(65.3)$ & $4(44.4)$ & 0.236 \\
\hline Blood urea nitrogen $(\mathrm{mg} / \mathrm{dL}$, mean $\pm \mathrm{SD})$ & $7.19 \pm 4.35$ & $10.89 \pm 2.17$ & 0.006 & $7.71 \pm 4.38$ & $9.29 \pm 3.42$ & 0.320 \\
\hline Systolic BP $(\mathrm{mm} \mathrm{Hg}$, mean \pm SD $)$ & $127.10 \pm 22.43$ & $97.33 \pm 28.95$ & $<0.001$ & $125.97 \pm 22.01$ & $93.55 \pm 33.65$ & $<0.001$ \\
\hline Diastolic BP $(\mathrm{mm} \mathrm{Hg}$, mean $\pm \mathrm{SD})$ & $75.08 \pm 12.04$ & $62.08 \pm 14.73$ & 0.002 & $74.65 \pm 11.46$ & $60.11 \pm 18.03$ & 0.002 \\
\hline Respiratory rate $(\mathrm{min}, \mathrm{mean} \pm \mathrm{SD})$ & $22.80 \pm 5.20$ & $29.50 \pm 4.68$ & $<0.001$ & $22.69 \pm 4.70$ & $32.33 \pm 3.87$ & $<0.001$ \\
\hline Shock index $($ mean $\pm S D)$ & $0.75 \pm 0.20$ & $1.17 \pm 0.59$ & $<0.001$ & $0.74 \pm 0.19$ & $1.37 \pm 0.55$ & $<0.001$ \\
\hline Confusion $(\%)$ & $2(4.3)$ & $8(66.7)$ & $<0.001$ & $6(12.2)$ & $4(44.4)$ & 0.019 \\
\hline Hospitalization (\%) & $29(63.0)$ & $12(100)$ & 0.012 & $33(67.3)$ & $8(88.9)$ & 0.192 \\
\hline Intensive care unit (\%) & $1 \quad(2.2)$ & $6(50.0)$ & $<0.001$ & $3(6.1)$ & $4(44.4)$ & 0.001 \\
\hline Mortality (28 days) (\%) & 0 & $4(33.3)$ & $<0.001$ & 0 & $4(44.4)$ & $<0.001$ \\
\hline Re-admission to hospital (28-day) (\%) & $4 \quad(8.7)$ & $3(25.0)$ & 0.123 & $6(12.2)$ & $1(11.1)$ & 0.924 \\
\hline CURB-65: confus & , serum urea nitr & $\begin{array}{l}\text { vel }>19.6 \mathrm{mg} / \mathrm{d} \\
\leq 60 \mathrm{~mm} \mathrm{Hg} \mathrm{di}\end{array}$ & iratory $\mathrm{r}$ & $\begin{array}{l}30 / \mathrm{min} \text {, low bloc } \\
5 \text { years. } \\
\text { d age } \geq 65 \text { years. }\end{array}$ & essure & \\
\hline
\end{tabular}


$p=0.044)$ and mortality was AUC 0.954 (0.899-0.999, $p=0.003)$. Figure 1 shows the ROC curve for predicting 4-week mortality.

Tables $4 \& 5$ shows ICU admission and death rates according to CURB and CRSI scoring systems. There are quite similar rates between the CURB and CRSI severe and non severe categories given in the tables.

Table 2 - Specificity, sensitivity, and positive and negative predictive values of CURB-65 and CRSI-65 indexes in predicting the need for intensive care unit after 28-day follow-up of 58 community-acquired pneumonia patients.

\begin{tabular}{lcc}
\hline Values & $\begin{array}{c}\text { CURB-65 } \\
(95 \% \mathrm{CI})\end{array}$ & $\begin{array}{c}\text { CRSI-65 } \\
(95 \% \mathrm{CI})\end{array}$ \\
\hline Sensitivity & 85.71 & 57.14 \\
& $(42.13$ to 99.64$)$ & $(18.41$ to 90.10$)$ \\
Specificity & 88.24 & 90.2 \\
& $(76.13$ to 95.56$)$ & $(78.59$ to 96.74$)$ \\
Positive predictive value & 50.0 & 44.44 \\
& $(30.79$ to 69.21$)$ & $(21.85$ to 69.59$)$ \\
Negative predictive value & 97.83 & 93.88 \\
& $(87.97$ to 99.64$)$ & $(86.64$ to 97.31$)$ \\
Area under curve & $0.870(0.710$ to 0.999$)$ & $0.737(0.506$ to \\
& 0.002 & $0.968)$ \\
P-value & \multicolumn{3}{c}{0.044} \\
\hline \multicolumn{2}{c}{ CRSI-65: confusion, respiratory rate, shock index-age $\geq 65$ years, } \\
CURB-65: confusion, serum urea nitrogen level, respiratory rate, blood \\
\multicolumn{2}{c}{ pressure and age $\geq 65$ years } \\
\hline
\end{tabular}

Table 3 - Specificity, sensitivity, and positive and negative predictive values of CURB-65 and CRSI-65 indexes in predicting mortality after 28-day follow-up of 58 community-acquired pneumonia patients.

\begin{tabular}{lcc}
\hline Values & $\begin{array}{c}\text { CURB-65 } \\
(95 \% \mathrm{CI})\end{array}$ & $\begin{array}{c}\text { CRSI-65 } \\
(95 \% \mathrm{CI})\end{array}$ \\
\hline Sensitivity & 100.0 & 100.0 \\
Specificity & $(37.96$ to 100.0$)$ & $(39.76$ to 100.0$)$ \\
Positive predictive value & 85.19 & 90.74 \\
& $(72.88$ to 93.88$)$ & $(79.70$ to 96.92$)$ \\
Negative predictive value & 33.33 & 44.44 \\
& $(20.87$ to 48.66$)$ & $(25.77$ to 64.84$)$ \\
Area under curve & 100.0 & 100.0 \\
$P$-value & 0.926 & $(59.94$ to 100.0$)$ \\
\hline
\end{tabular}

CRSI-65: confusion, respiratory rate, shock index-age $\geq 65$ years, CURB-65: confusion, serum urea nitrogen level, respiratory rate, blood pressure and age $\geq 65$ years
The rates shown in these tables are in line with the results obtained by McNemar and Kappa analyzes, indicating that the CRSI index can be used instead of CURB. Kappa measure of agreement was calculated 2 scoring system. Strength of the kappa coefficients were substantial (kappa 0.695).

Discussion. In the present study, we determined that the CRSI-65 score can be used in predicting 4-week mortality and the need for intensive care in cases of severe CAP (CRSI-65 score $\geq 3$ ). The new scoring method proposed is based on patients' vital signs and clinical characteristics. No laboratory tests are needed to calculate the CRSI-65 score. Owing to this feature, the CRSI-65 can provide a practical approach for prehospital teams, emergency room triage, and outpatient treatment management. In primary care, it may be useful in referring patients followed by family medicine centers to hospitals.

The most important decision in managing CAP is predicting mortality and admitting a high-risk patient to the hospital. Other important decision is hospitalization and admission to intensive care unit. The decision is either hospitalization or admission to the intensive care unit, which is still a clinical decision in CAP management; however, the scoring systems may help clinicians in taking a better decision. ${ }^{3}$ For this reason, our study focused on patients with severe CAP. The CRSI-65 score was performed similarly as the CURB-65 score in predicting 4 -week mortality (95\%

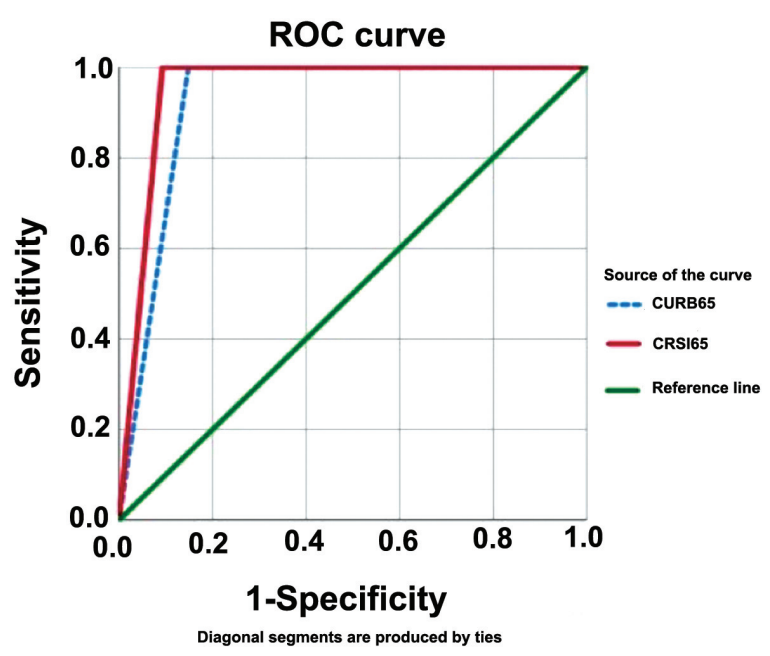

Figure 1 - Receiver operating characteristic curve for confusion, serum urea nitrogen level, respiratory rate, blood pressure and age $\geq 65$ years (CURB-65) and confusion, respiratory rate, shock index-age $\geq 65$ years (CRSI-65) indexes for predicting 28-day mortality. 
Table 4 - Intensive care unit (ICU) admission rate according to CURB and CRSI scoring systems.

\begin{tabular}{llr}
\hline Scoring systems & \multicolumn{2}{c}{ ICU admission } \\
& Yes $(\mathrm{n}=7)$ & No $(\mathrm{n}=51)$ \\
\hline CURB & & \\
Severe $(\mathrm{n}=12)$ & $6(50.0)$ & $6(50.0)$ \\
Non severe $(\mathrm{n}=46)$ & $1(2.17)$ & $45(97.8)$ \\
CRSI & & \\
Severe $(\mathrm{n}=9)$ & $4(44.4)$ & $5(55.6)$ \\
Non severe $(\mathrm{n}=49)$ & $3(6.1)$ & $46(93.9)$ \\
\hline
\end{tabular}

CRSI-65: confusion, respiratory rate, shock index-age $\geq 65$ years, CURB-65: confusion, serum urea nitrogen level, respiratory rate, blood pressure and age $\geq 65$ years
CI, AUC ROC $=0.954[0.899-0.999], p=0.003$ ). The CRSI-65 score was weaker than the CURB-65 in predicting the 4-week need for intensive care; however there was a statistically significant relationship $(95 \%$ $\mathrm{CI}, \mathrm{AUC}=0.737[0.506-0.968], p=0.044)$.

One of the improvements in the CRSI-65 score we recommend is the inclusion of the SI in the scoring system instead of the blood pressure parameter. This will reduce the risk of false negatives in hypertensive patients. The scoring system also includes the evaluation of heart rate, which is another physiological parameter and a component of the SI. The SI is a physiological parameter that has been shown to be prognostically useful in many diseases, and there is evidence showing that it can be used in CAP. ${ }^{10,13,14}$ Using the SI, which is the ratio of heart rate to systolic blood pressure, instead of the blood pressure parameter may be a better physiologic indicator. ${ }^{13}$ Sankaran et $\mathrm{al}^{13}$ investigated the relationship between the SI and CAP and reported that a SI of $\geq 1$ could be used to predict 4-6-week mortality of patients with CAP. ${ }^{10}$ In another study, wherein the SI was included, the index value was significantly higher in patients with severe CAP than in patients without severe CAP $(p=0.0001)$. In the present study, no adjustments were made in the calculations related to fever or hypertension, which could have affected the SI, or drug use (namely, beta blockers), which could have affected blood pressure and flow rate. Nevertheless, the SI values were statistically significant for both the CURB-65 and CRSI-65 scores in the groups with and without severe CAP $(p<0.001, p<0.001)$. Therefore, the index is useful irrespective of the factors that can potentially affect the SI. ${ }^{13}$

The second improvement in the CRSI-65 score is the removal of blood urea nitrogen (BUN) from the scoring system. It has been suggested that CRB-65 score can be used in outpatients instead of the CURB-65
Table 5 - Death rate according to CURB and CRSI scoring systems.

\begin{tabular}{|c|c|c|}
\hline \multirow[t]{2}{*}{ Scoring systems } & \multicolumn{2}{|c|}{ Death } \\
\hline & Yes $(n=4)$ & No $(n=54)$ \\
\hline \multicolumn{3}{|l|}{ CURB } \\
\hline Severe $(n=12)$ & $4(33.3)$ & $8(66.7)$ \\
\hline Non severe $(n=46)$ & $\begin{array}{ll}0 & (0)\end{array}$ & $46(100)$ \\
\hline \multicolumn{3}{|l|}{ CRSI } \\
\hline Severe $(n=9)$ & $4(44.4)$ & $5(55.6)$ \\
\hline Non severe $(n=49)$ & $\begin{array}{ll}0 & (0)\end{array}$ & $49(100)$ \\
\hline
\end{tabular}

CRSI-65: confusion, respiratory rate, shock index-age $\geq 65$ years, CURB-65: confusion, serum urea nitrogen level, respiratory rate, blood pressure and age $\geq 65$ years

score because the CURB-65 score leads to the difficulty in scoring BUN in outpatients. ${ }^{4,12}$ The CRB-65 score obtained by removing BUN shows results surprisingly similar to those of the CURB-65 score. ${ }^{15} \mathrm{~A}$ study by Ochoa-Gondar et $\mathrm{al}^{16}$ reported that CRB-65 was performed as well as, or better than, the CURB-65 in all categories. There are other studies that question whether BUN is a mandatory parameter in the severity scoring of CAP patients. ${ }^{17,18}$ In the present study, BUN was significantly higher in the group with severe CAP according to the CURB-65 score $(p=0.006)$. However, there was no statistically significant difference in BUN in the group with severe CAP according to the CRSI-65 score $(p=0.320)$. It seems that this difference in BUN, combined with other parameters in the scoring, did not affect mortality predictions.

The scoring system most similar to the CRSI-65 score was first reported by Curtain et $\mathrm{al}^{7}$ as the CRSI score without the age parameter. Curtain et $\mathrm{al}^{7}$ considered patients with a CRSI score of $\geq 2$ to have severe CAP. For predicting 6-week mortality, they reported that the CRSI score had a specificity of $83.9 \%$ (74.1-90.6), sensitivity of $75 \%$ (35.6-95.5), positive predictive value of $30 \%(12.8-54.3)$, and negative predictive value of $97.3 \%(89.8-99.5)$ at $95 \%$ CI. $^{7}$ These results are in accordance with our findings; however, our results provide better specificity and negative predictive value. It is very important to safely and reliably predict 4-6 week mortality in patients with CAP. In this respect, our results indicate that the CRSI-65 score may be useful in emergency department triage and outpatient follow-up.

Another issue that should be mentioned is the age parameter used in the CAP severity scoring. Age parameter increases false positivity rate in elderly patients and leads to false negativity in younger patients. We believe that the age parameter should be used in CAP severity scoring owing to the evidence showing 
a correlation between increasing age and increasing mortality in patients with CAP and the increasing comorbid conditions with increasing age that lead to complex conditions. ${ }^{19,20}$ However, if age is the only criterion, it is not inappropriate to evaluate patients as being at risk in the absence of other criteria, and close monitoring of the patient seems to be a better approach. ${ }^{16}$

Study limitations. Our study included patients who were admitted to the emergency department. Although the patients had a low risk score, their admission to the emergency department may have affected the clinician's follow-up and treatment plan. In fact, the rate of hospitalization was high in this study (70.7\%). We did not investigate underlying respiratory conditions, such as chronic obstructive pulmonary disease (COPD), but it has been previously reported that COPD does not affect mortality in CAP. ${ }^{13,21}$ This study was conducted in a single center. The general characteristics of hospitals, their patient population and the characteristics of the population served tend to vary; thus, the study needs to be generalized.

In conclusion, the CRSI- 65 score provides remarkable results, indicating that it can be used as a severity score in patients with CAP and can guide mortality prediction. The CRSI-65 score, which includes the SI, can provide a useful and practical predictive tool in primary care and in emergency or pre-hospital settings where urea value cannot be measured, and hence, it can accelerate the initiation of treatment. The CRSI-65 score provides potential support for our hypothesis that mortality can be predicted in CAP without laboratory testing. However, there is a need for further studies in different populations with larger patient groups.

Acknowledgment. The authors gratefully acknowledge Enago (www.enago.com.tr) for English language editing.

\section{References}

1. Nullmann H, Pflug MA, Wesemann T, Heppner HJ, Pientka L, Thiem U. External validation of the CURSI criteria (confusion, urea, respiratory rate and shock index) in adults hospitalised for community-acquired pneumonia. BMC Infect Dis 2014; 14: 39.

2. Kosar F, Alici DE, Hacibedel B, Arpinar Yigitbas B, Golabi P, Cuhadaroglu C. Burden of in adults over $18 \mathrm{y}$ of age. Hum Vaccin Immunother 2017; 13: 1673-1680.

3. Frenzen FS, Kutschan U, Meiswinkel N, Schulte-Hubbert B, Ewig S, Kolditz M. Admission lactate predicts poor prognosis independently of the CRB/CURB-65 scores in communityacquired pneumonia. Clin Microbiol Infect 2018; 24: e306-e3111.

4. Woodhead M, Blasi F, Ewig S, Garau J, Huchon G, Ieven M et al. Guidelines for the management of adult lower respiratory tract infections. Clin Microbiol Infect 2005; 26: 1138-1180.
5. Mandell LA, Wunderink RG, Anzueto A, Bartlett JG, Campbell GD, Dean NC et al. Infectious Diseases Society of America/American Thoracic Society consensus guidelines on the management of community-acquired pneumonia in adults. Clin Infect Dis 2007; 44 (Suppl 2): 27-72.

6. Sharp AL, Jones JP, Wu I, Huynh D, Kocher KE, Shah NR et al. CURB-65 performance among admitted and discharged emergency department patients with community-acquired pneumonia. Acad Emerg Med 2016; 23: 400-405.

7. Curtain JP, Sankaran P, Kamath AV, Myint PK. The usefulness of confusion, urea, respiratory rate, and shock index or adjusted shock index criteria in predicting combined mortality and/or ICU admission compared to CURB-65 in community-acquired pneumonia. Biomed Res Int 2013; 2013: 1-6.

8. King RW, Plewa MC, Buderer NMF, Knotts FB. Shock index as a marker for significant injury in trauma patients. Acad Emerg Med 1996; 3: 1041-1045.

9. Bauer TT, Ewig S, Marre R, Suttorp N, Welte T, CAPNETZ Study Group. CRB-65 predicts death from communityacquired pneumonia. J Intern Med 2006; 260: 93-101.

10. Sankaran P, Kamath AV, Tariq SM, Ruffell H, Smith AC, Prentice $P$, et al. Are shock index and adjusted shock index useful in predicting mortality and length of stay in communityacquired pneumonia? Eur J Intern Med 2011; 22: 282-285.

11. Ewig S, de Roux A, Bauer T, García E, Mensa J, Niederman M. Validation of predictive rules and indices of severity for community acquired pneumonia. Thorax 2004; 59: 421-427.

12. Lim WS, van der Eerden MM, Laing R, Boersma WG, Karalus N, Town GI, et al. Defining community acquired pneumonia severity on presentation to hospital: an international derivation and validation study. Thorax 2003; 58: 377-382.

13. Myint PK, Musonda P, Sankaran P, Subramanian DN, Ruffell H, Smith AC, et al. Confusion, Urea, Respiratory Rate and Shock Index or Adjusted Shock Index (CURSI or CURASI) criteria predict mortality in community-acquired pneumonia. Eur J Intern Med 2010; 21: 429-433.

14. Schmidt T, Lassen A, Wiil UK. Occurrence of elevated shock index in acute patients and development of a proportional shock index metric. Conf Proc IEEE Eng Med Biol Soc 2016; 2016: 2411-2414.

15. Ewig S, Welte T. CRB-65 for the assessment of pneumonia severity: who could ask for more? Thorax 2008; 63: 665-666.

16. Ochoa-Gondar O, Vila-Corcoles A, Rodriguez-Blanco T, Ramos F, de Diego C, Salsench E, et al. Comparison of three predictive rules for assessing severity in elderly patients with CAP. Int J Clin Pract 2011; 65: 1165-1172.

17. Ugajin M, Yamaki K, Iwamura N, Yagi T, Asano T. Blood urea nitrogen to serum albumin ratio independently predicts mortality and severity of community-acquired pneumonia. Int J Gen Med 2012; 5: 583-589.

18. Long B, Long D, Koyfman A. Emergency medicine evaluation of community-acquired pneumonia: History, examination, imaging and laboratory assessment, and risk scores. J Emerg Med 2017; 53: 642-652.

19. Zhang ZX, Yong Y, Tan WC, Shen L, Ng HS, Fong KY. Prognostic factors for mortality due to pneumonia among adults from different age groups in Singapore and mortality predictions based on PSI and CURB-65. Singapore Med J 2018; 59: 190-198.

20. Parsonage M, Nathwani D, Davey P, Barlow G. Evaluation of the performance of CURB-65 with increasing age. Clin Microbiol Infect 2009; 15: 858-864.

21. Snijders D, van der Eerden M, de Graaff C, Boersma W. The influence of COPD on mortality and severity scoring in community-acquired pneumonia. Respiration 2010; 79: 46-53. 